\title{
Assessments of Community Mental Health Support Systems: A Key Informant Approach
}

\author{
Joseph P. Morrissey, Ph.D. \\ M. Susan Ridgely, M.S.W. \\ Howard H. Goldman, M.D., Ph.D., M.P.H. \\ W. Todd Bartko, Ph.D.
}

\begin{abstract}
This article describes the development of a 'key informant survey' to assess the performance of local systems of care for persons with a chronic mental illness. The measure yields ratings of: (1) the extent to which clients experience service delivery problems in 11 community support system elements, (2) overall performance of the community support system, and (3) the performance of local mental health authorities. Following pre-testing, the survey was administered to 699 respondents in nine U.S. cities. Internal consistency coefficients were found to be within acceptable ranges for all of the scales across all nine cities. Analyses comparing mean values for performance ratings showed that the nine sites could be arranged into three groups representing high, medium and low system performance. These findings support observations from site visits conducted over several years and suggest that the survey is a valid instrument for assessing local systems of care.
\end{abstract}

Joseph P. Morrissey, Ph.D., is Professor of Social Medicine and Deputy Director for Research, Cecil G. Sheps Center for Health Services, University of North Carolina at Chapel Hill, 725 Airport Road Suite 210, Chapel Hill, NC 27599-7590. Susan Ridgely, M.S.W., is Research Associate and Associate Director, Center for Mental Health Services Research, Department of Psychiatry, University of Maryland at Baltimore. Howard H. Goldman, M.D., Ph.D., M.P.H., is Professor and Director, Center for Mental Health Services Research, Department of Psychiatry, University of Maryland at Baltimore. W. Todd Bartko, Ph.D., is a Fellow at the Center for Human Growth and Development, University of Michigan at Ann Arbor.

This research was supported by grants from the Robert Wood Johnson Foundation (12558) and the National Institute of Mental Health (MH44839). We would like to thank Clara Muschkin and Deborah F. Jacobs for their assistance in data organization and analysis. 
As the view of providing for the needs of chronically mentally ill persons has broadened beyond "treatment" to ensuring that basic life needs such as those for food and shelter are met, the view of what constitutes the mental health service system has also broadened. Rather than the traditional mental health service delivery system, the concept of a 'community support system' (CSS) is central to current program development efforts (Turner and TenHoor, 1978; Tessler and Goldman, 1982). This concept encompasses a much more complex reality for researchers and service providers alike due to the sheer number and types of providers (e.g., mental health, social welfare, employment, housing, rehabilitation, and criminal justice) operating in the system of care.

Recent intervention efforts have focused on improving community support systems within this new framework. The Robert Wood Johnson Foundation Program on Chronic Mental Illness was designed to create systemic changes in the organization, financing and delivery of mental health and other support services in nine U.S. cities (Aiken, Somers \& Shore, 1987; Shore \& Cohen, 1990). The primary goal of the demonstration was to improve the coordination of services to persons with a chronic mental illness by creating a central mental health authority at the local level as the main organizational intervention at each site (Goldman, Morrissey \& Ridgely, 1990). The local mental health authorities were intended to assume responsibility for meeting the treatment, housing and supportive care needs of these persons. As part of a comprehensive evaluation of this demonstration (Goldman et al., 1990), we sought to determine how successful the nine cities were in improving the systems of care for persons with a chronic mental illness.

Whereas systems concepts for describing service delivery (such as availability, accessibility, accountability, adequacy, quality, continuity, comprehensiveness, and viability) have become well-accepted in the mental health services arena, survey methods for assessing the capacity and performance of service systems for chronically mentally ill persons are not well developed. After looking for an established instrument without success, we developed a 'key informant survey' to obtain performance ratings of local service systems from knowledgeable persons at each of the demonstration sites. The idea of expert opinion polling has a long tradition in the mental health needs assessment literature (Attkisson et al., 1978). We adopted this strategy to obtain performance ratings on how well local communities were meeting the needs of CMI persons living in the demonstration sites. We also hoped to develop an instrument which could readily be used in any commu- 
nity to characterize the functioning of the local community support system.

\section{QUESTIONNAIRE DEVELOPMENT}

Our approach to the development of a new instrument was informed by a number of sources. We began with the concept of a community support system and attempted to determine what services are subsumed under this heading. The 'official' NIMH listing of CSS elements was most helpful in delineating the range and types of community-based services needed by persons with a chronic mental illness (Stroul, 1988). These services are not limited to mental health treatment but also include, for example, client identification and outreach, crisis response services, and advocacy.

Second, we reviewed the goals of the RWJF Program on Mental Illness, as reflected in application materials (PMI, 1986) and associated published reports (Aiken, Somers \& Shore, 1986), to identify the relevant domains and dimensions of the interventions. The goals of the demonstration were to: (1) develop a local mental health authority to be given central responsibility (administrative, clinical, and fiscal) for delivering care to persons with a chronic mental illness; (2) ensure continuity of care in the service system by assigning a staff member or team responsibility for each client; (3) provide a full range of services to persons with a chronic mental illness; (4) establish a housing program to expand the number, variety, and quality of community-based housing units, and (5) devise a financing strategy to support the system of care by combining state, county, and city funds into a single stream and generating new sources of revenue. Clearly, our evaluation needed to assess the extent to which each of these goals were accomplished in each of the cities.

Third, we looked to the available literature for instruments to measure service system capacity and performance and for characterizations of well-developed service 'systems' that would help us construct items for inclusion in a questionnaire (see, for example, Mechanic \& Aiken, 1987; Walsh \& Leigland, 1986; Dickey \& Goldman, 1986). We adapted the instrument that had been used in an earlier study of CSS programs in New York State (Morrissey, Tausig \& Lindsey, 1985: 120-121; 1986). In that study, respondents were asked to use a Likert-type scale to rate the extent to which thirteen service delivery problems encountered by persons with a chronic mental illness were occuring in their local 
community. The range of items was quite limited, however, and there was no reference to local mental health authorities in the list of items rated. Two other instruments were also helpful. First, the "Denver CMI Initiative: Key Informant and Attitude Survey" (Wilson et al., 1988) influenced our thinking on how to ask about specific service delivery problems. We used some of these questions, added others and rearranged the questions along specific categories of service. Second, the work of Grusky and Tierney (1988) influenced the development of our section on service system performance.

Finally, we developed a template for conducting site visits to each of the nine cities. The site visits included interviews with project administrators, clinicians, clients, advocates, family members, and government officials by two or three evaluation team members. These visits were conducted prior to and following the planning phase of the demonstration and again near the mid-point of the implementation period. The qualitative information gathered during these visits was obtained using rigorous procedures such as reinterviewing informants, debriefing in the field among site visitors, verification of factual material, and sharing of observations with external observers (Silverman, Ricci \& Gunter, 1990). The purpose of these visits was to provide us with an initial view of the systems of care through the eyes of local stakeholders and a range of ideas about the ingredients and criteria for service system performance (Goldman, Morrissey \& Ridgely, 1990). Following procedures for linking qualitative and quantitative data (Sieber, 1973), these ideas were incorporated into the questionnaire in terms of both the structure of the survey and the content of structured and unstructured questions.

\section{CONTENT OF THE KEY INFORMANT SURVEY}

The key informant survey questionnaire, Assessing Local Service Delivery Systems for Chronically Mentally Ill Persons, yielded both quantitative and qualitative data. Qualitative observations were obtained in the form of several open-ended questions about the major accomplishments and shortcomings of the local RWJF demonstration. These questions were included so that responses could be incorporated into our analyses whenever possible to illustrate aspects of the various service systems or differences in the perceptions of various groups of respondents. 
The quantitative data are derived from a series of Likert-type scale items that relate to several distinct constructs. Simple additive scales were constructed for each measure. All composite indices were rescaled to the original five point Likert scoring in which 1 indicates the lowest performance and 5 indicates the highest or most positive performance.

\section{Service Problems}

Several scales derived from this questionnaire probed the types of problems encountered with regard to the eleven community support system (CSS) service elements. These elements depart slightly from the 'official' NIMH listing which includes services for outreach, mental health treatment, health, crisis response, housing, basic needs, case management, rehabilitation, peer support, family and community support, and advocacy (Stroul, 1988). Basically, we made four alterations to insure that the range of services being developed at the RWJF demonstration sites could be easily rated. We split the CSS element 'rehabilitation' into two components, 'psychosocial' and 'prevocational and vocational'; we added 'substance abuse services' as a separate category in recognition that a significant number of chronically mentally ill persons also have substance abuse problems; we grouped 'peer support' and 'family support' into a single category called 'other support'; and we dropped the protection and advocacy category as this specific form of legal advocacy was not a priority at any site.

These scales were designed to provide an indication of client needs or the extent to which persons with a chronic mental illness were experiencing service delivery problems in July 1989 vs. two years earlier at the start of the demonstration. This is the only retrospective information requested in the questionnaire. The purpose of these questions was to provide some indication of the nature of the problems encountered by CMI persons in each city, and the extent to which the problems were increasing or abating. We had expected some difficulty with the reliability of this information, due to incomplete recall, as well as in responses from persons with a relatively brief tenure in their positions. Although these problems do occur (manifest in the higher proportions of missing data for variables in these scales), the data are useful for some comparisons. Certainly a more adequate source for across-time comparisons will be provided in the Time 2 survey, which will include all or most of the items comprising the Time 1 survey 
questionnaire. Thus, except for the retrospective questions, the data are considered cross-sectional and subject primarily to descriptive and exploratory analysis.

\section{Overall System Performance}

Four scales measuring the overall performance of the community support system for persons with a serious and persistent mental illness were developed for this study. Adequacy of services, or the extent to which persons needing each of the CSS services actually receive them, is the first dimension of system performance. The respondent is asked "How many CMI persons who need this service are getting it?" The same eleven CSS services included in the previous section on service problems are listed with brief definitions and the respondent rates each service according to a five point "very good" to "very poor" scale. Responses are summed to reflect the adequacy of services across the eleven CSS categories. Quality of services, the extent to which each of the CSS services meet current professional standards on interpersonal, technical and physical location considerations, is the second dimension of overall service system performance. The respondent is asked "How would you rate the quality of care provided to CMI persons in each of the following services?" The eleven CSS services are listed and the respondent rates each service according to a five point "very good" to "very poor" scale. Availability of services, the extent to which needed CSS services actually exist, is the third dimension of overall system performance. The respondent is asked, "How well does the current service system for chronically mentally ill persons perform in the following activities?" Issues assessed include 'avoiding waiting lists', 'reducing red tape', and 'providing transportation' (see Table 1).

Respondents rate each item on a five point scale from "very well" to "very poorly."

The fourth dimension of overall system performance is coordination of services, or the extent to which agencies do things in a concerted way. The stimulus question is identical to that used in the section on availability of services. Here, however, the system performance characteristics assessed include the 'sharing of information between agencies', 'elimination of conflicting requirements', and 'avoiding duplication of effort' (see Table 1). Respondents rate each item on a five point scale from "very well" to "very poorly." 
Joseph P. Morrissey, Ph.D., et al.

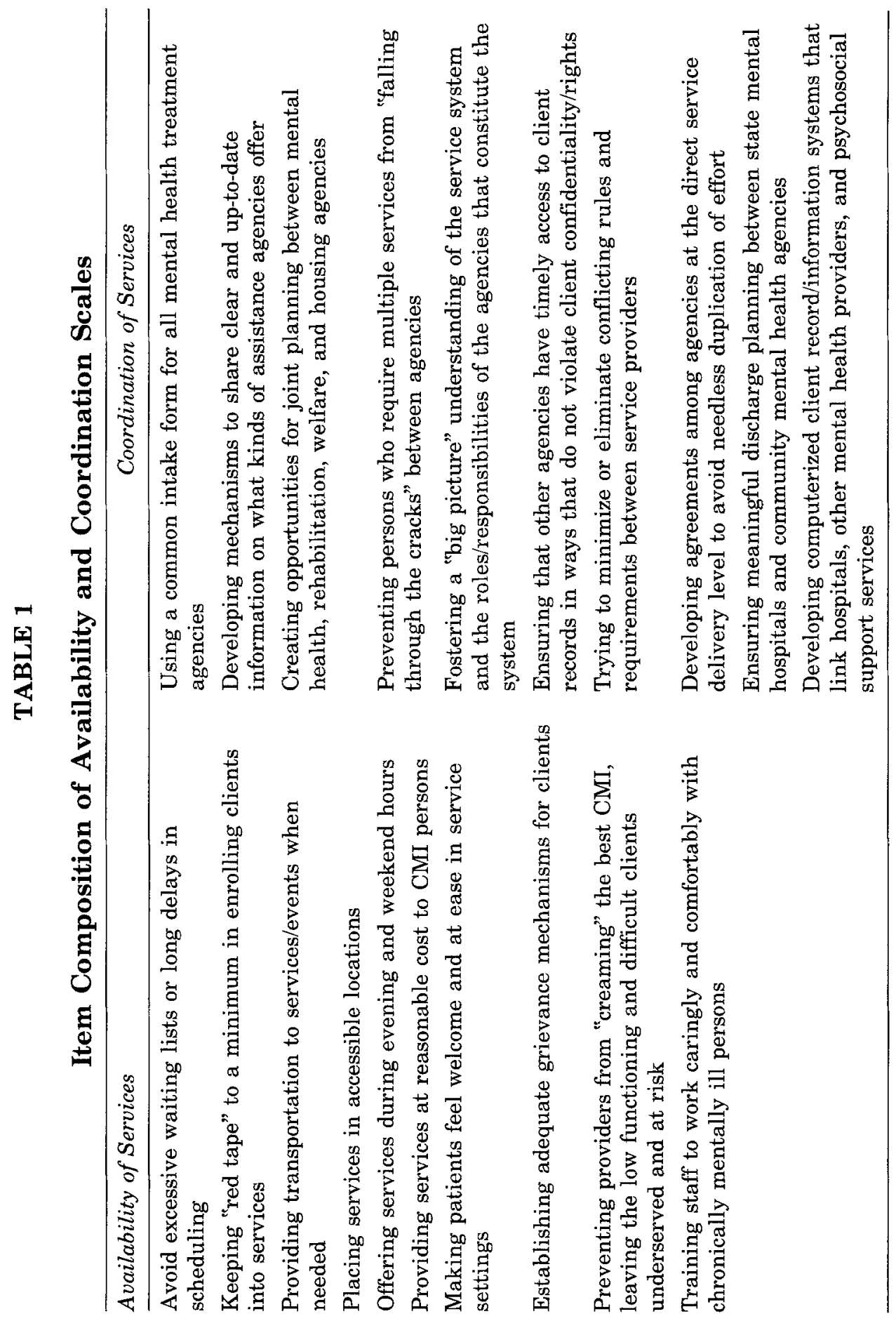




\section{Mental Health Authority Performance}

In addition to ratings of overall system performance, three scales measuring the performance of the mental health authorities were constructed. Table 2 shows the items included in each of the following fouritem scales: (1) Administrative authority, reflected the extent to which the mental health authorities were centralizing the administration of the service system, (2) Clinical authority, the extent to which the mental health authorities had assured the provision of appropriate clinical services for clients, and (3) Fiscal authority, the extent to which the mental health authorities had developed a fiscally-sound plan for funding services for clients. A 1-5 Likert scale was used for these scales.

For each of the items measuring mental health authority performance, respondents are asked, "To what extent do you agree with the following statements about the (local mental health authority)?" Respondents rate each issue on a five point scale from "strongly agree" to "strongly disagree." The internal consistency coefficients (Cronbach's alpha) for each of the scales are presented in Table 3.

The key informant survey originally included several additional items concerning the functioning of the mental health authorities. Many respondents, however, were not familiar with detailed operational workings of the authority's financing, monitoring, and management practices. Consequently, this led to many respondents being unable to answer these questions. It also speaks to one of the limitations of a key informant approach to systems assessment. Namely, the logic of a survey of this type assumes that respondents are familiar with the issues raised. Here, however, many aspects of mental health authority performance are hidden to all but those most closely associated with the authority. Other strategies of data collection need to be considered to assess these practices.

\section{Pretesting}

The key informant survey was pretested in Rochester, New York, in collaboration with the Monroe-Livingston Demonstration Project. This site was chosen because the demonstration project, being operated by Integrated Mental Health, Inc., was a systems demonstration similar to, but not a part of, the RWJF Program (Babigian and Marshall, 1989). While the focus of the reorganization in Rochester had to do with a capitation financing demonstration, Integrated Mental Health, Inc. acted as a local mental health authority, managing and coordinating 


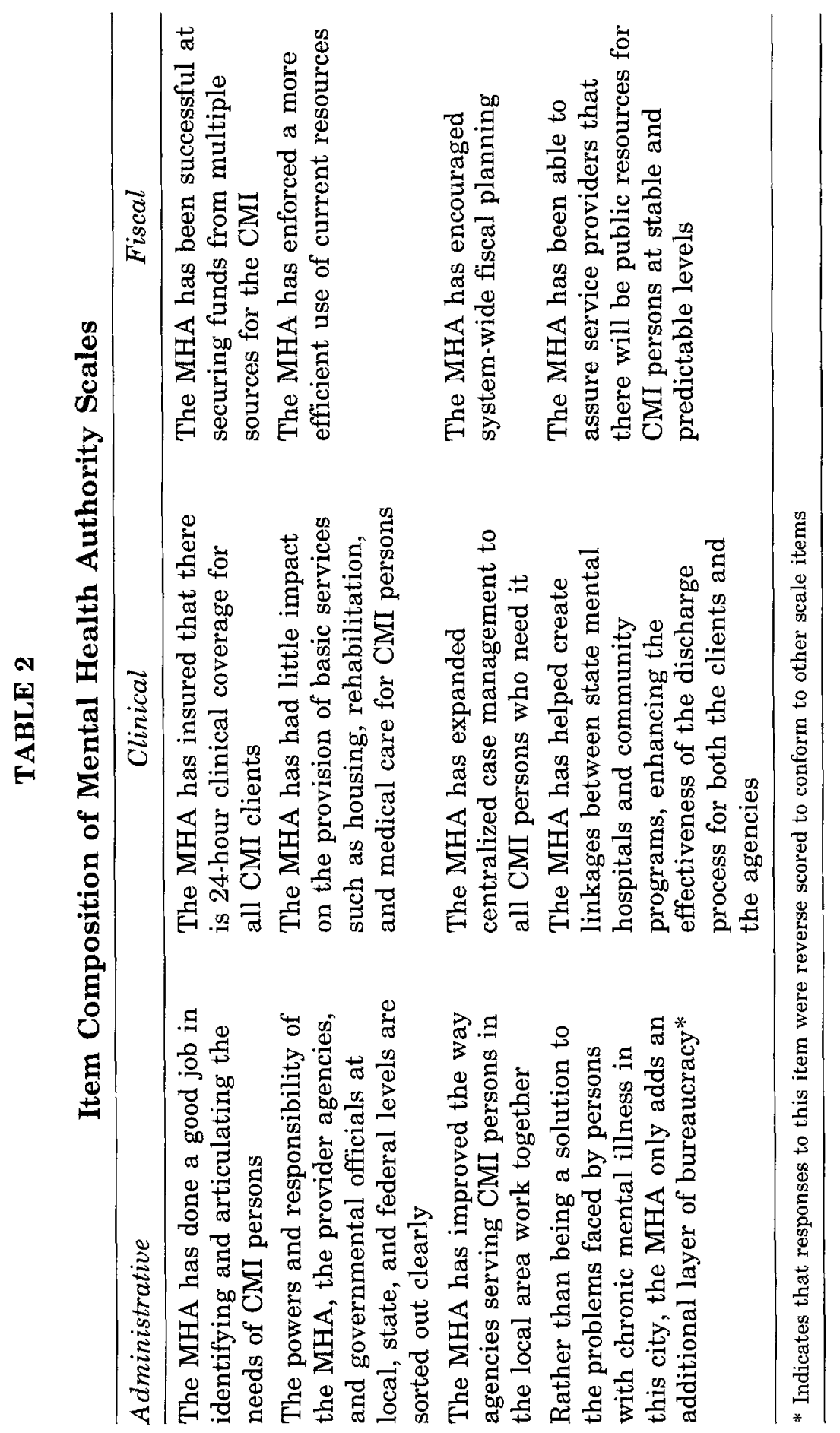




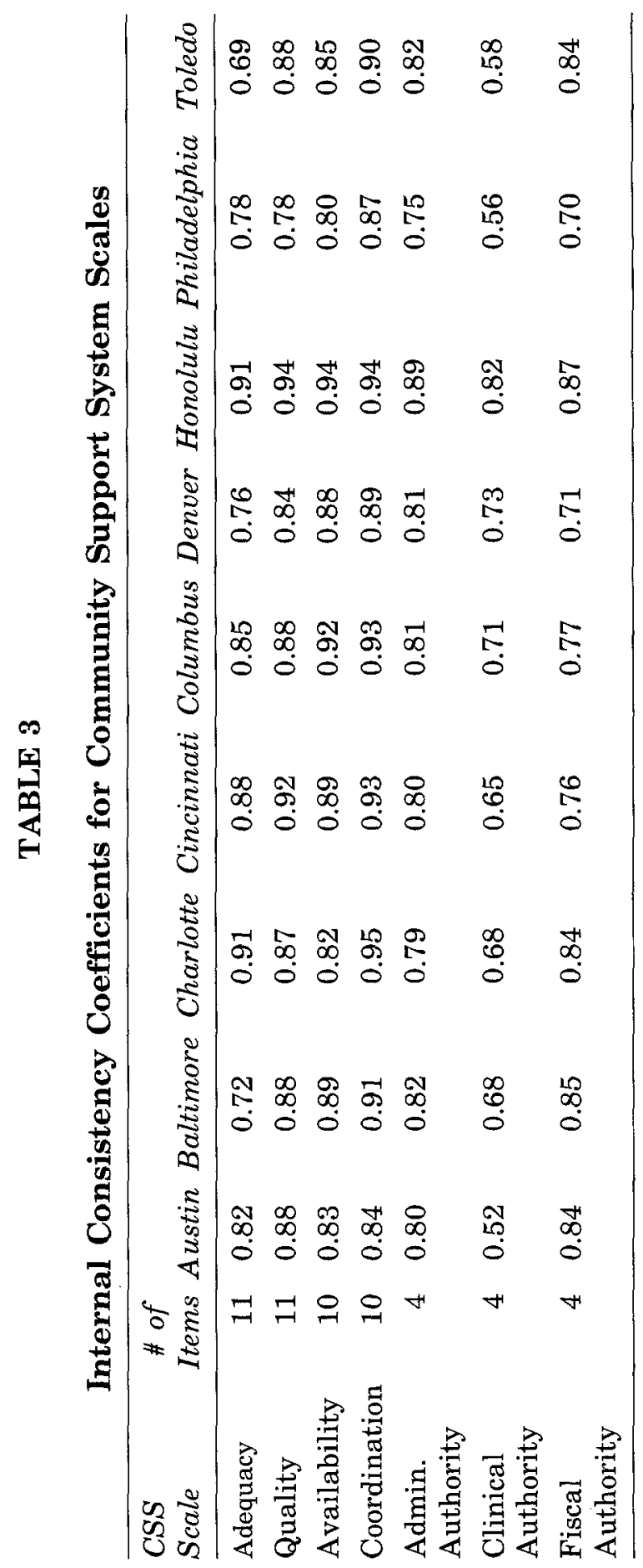


services much as do the authorities in the RWJF sites. The pretest indicated that the key informant instrument had face validity to participants in the various sectors and could be administered as a mailed questionnaire with an acceptable response rate after two follow-ups.

\section{Respondent Sample}

The respondent pool for the present study was developed in consultation with the RWJF Program staff at each of the nine RWJ/PCMI sites. The process was iterative. We relied upon the concept of a community support system and its associated functions to identify the sectors from which knowledgeable respondents could be located. In each sector, we were especially interested in agencies that were providing services to persons with chronic mental illnesses. Additionally, we were also interested in the views of other persons (advocates, officials, interested citizens) who were familiar with the activities of the demonstration projects. For most of these agencies the key informant was to be the CEO, but for some more centrally involved agencies, multiple respondents were sought. One of the reasons to seek multiple points of view within an agency is that issues may be differentially perceived depending on one's place in the agency (i.e., the 'emergency room' versus the 'board room'). We reviewed the lists created by the Program staff, adding and substituting agencies and informants where appropriate. We sought to apply the same selection criteria and sampling strategy in each city (regardless of the boundaries of the system as viewed by local participants) so that cross-city comparisons would be meaningful.

One of the difficulties we encountered in creating these lists was the fact that our view of the service system was broader than the view of the RWJF Project staff in some of the sites. When asked to list the names of key stakeholders in services outside of traditional mental health treatment services (e.g., jails, police, the media) project staff often had difficulty doing so. We, therefore, relied on information from our site visits to ensure that all relevant agencies were included in our survey.

\section{Data Collection}

Following sample selection, survey questionnaires were mailed to the key informants at each site. The data collection process began in June, 1989 for five of the cities (Baltimore, Denver, Columbus, Cincinnati, and Toledo) which were further along in program implementation. 
Mailings to the other four cities (Austin, Charlotte, Honolulu, and Philadelphia) were initiated in September 1989 and data collection extended through early December.

In each city, the original list of respondents was supplemented with community leaders identified through a snowball sampling procedure. This sample was obtained by asking the initial key informants to list up to 15 persons who they considered were making important positive contributions to the city's service system for the chronically mentally ill. The snowball sample proved to be a useful means of identifying additional key persons in the mental health service systems in each city. In addition, the snowball sample provided a means of checking the validity of the procedure used to obtain the original sample as well as the representativeness of the sample with regard to mental health leaders (both professional and lay) at each site. In fact, the snowball samples and the original samples proved to be remarkably consistent, with an overlap of about seventy-five percent in each city. The remaining names obtained from the snowball sample were then added to the mailing lists for each site; these respondents received questionnaires identical to those sent to the original sample.

In this way, questionnaires were mailed to between 86 and 172 respondents in each city. Two follow-up mailings were carried out in each city, as well as telephone prompts when necessary. Final response rates across cities varied from 59 to 73 percent.

\section{Respondents and Nonrespondents}

The sample in each city was designed to include persons with diverse roles and with diverse affiliations within the community support system. The distribution of the total sample across role and affiliation categories varied considerably across sites, in accordance with the particularities of the local service systems. In this section we will present some general observations regarding the characteristics of respondents and their representativeness vis-a-vis the total sample.

The total sample was comprised largely of persons in the roles of board member, agency director, and program director. The percentage of persons in the sample who were included in these three role categories ranged from 58 percent in Denver to 85 percent in Toledo. In the remaining cities, these categories made up approximately two-thirds of the sample. The response rate varied considerably across role categories in most cities. Board members of the mental health authority and elected officials were consistently underrepresented in our respondent 
group due to a response rate of less than 50 percent. The exception to this was Toledo, where members responded at a rate of almost 75 percent, equal to that of agency and program directors.

With regard to the respondents' area of affiliation, the distribution across categories in the total sample was more uniform than was the case with the role distributions. Most respondents were affiliated with the mental health area (i.e., either the mental health authority, State or other psychiatric hospital, Community Mental Health Center (CMHC), or another type of mental health agency). This segment ranged from 49 percent in Charlotte to 68 percent in Baltimore; the other cities hover around the 60 percent mark. Within the mental health area, the response rate was lowest among persons affiliated with the mental health authority boards and with CMHCs, the two largest segments. All members of the 10-25 person boards were surveyed, but in many instances these community leaders and influential lay persons indicated that they lacked first-hand knowledge about the workings of the community support system. The mental health authority was consistently underrepresented (more so for board members than for staff) in all cities except Toledo and Honolulu.

The distributions of respondents and nonrespondents across cities have important implications for the representativeness and replicability of the samples. With regard to representativeness, we are generally satisfied with the distribution across respondent categories even though the overall response rate was less than we anticipated. Further, our analyses lead us to believe that the respondent groups are adequately representative of the target sample of persons in key positions for evaluating community support service systems.

\section{DISCUSSION}

The key informant survey was developed to evaluate the RWJ/PCMI demonstration but it can be adapted and used for other cities and settings as well. (The Key Informant Survey has been adapted for use in assessing children's mental health services in an ongoing study in North Carolina. Copies of both the adult and child versions of the instrument are available upon request from the first author.) The design of the present evaluation (Goldman et al, 1990) relies upon multiple sources of data and a strategy of triangulation to assess system performances and outcomes. These multiple sources of data help to 
compensate for limitations or biases associated with each source or method of data collection.

The key informant survey is designed to obtain the ratings of various stakeholder groups about the functioning of the local community support system. The other sources of data we have collected are based on site visits and an interorganizational network analysis.

Findings from the key informant survey data collected in 1989 are consistent with observations derived from our annual site visits (Goldman, in press; Goldman, Morrissey, \& Ridgely, 1990). For example, respondents' ratings of the performance of the local systems of care revealed that the cities can be sorted into three groupings (see Morrissey et al, 1992). Toledo, Columbus, Cincinnati, and Charlotte generally had the highest ratings of service system functioning. Austin, Denver, and Honolulu fell into the middle group. The lower group on the performance scales included Baltimore and Philadelphia. This ranking corresponds to what we have learned about program implementation from our site visiting. The four cities with the highest ratings, for example, already had a pre-existing mental health authority and were expected to proceed with service enhancements more rapidly than the other cities. Further, we observed during our visits that the largest cities (Baltimore and Philadelphia) both started with highly fragmented community support systems and this situation is reflected in the lower ratings for these sites. Finally, preliminary results from our interorganizational network analysis using data collected in six of the cities appear to support our site visit observations as well as the findings from the key informant survey (Morrissey et al, 1992).

In sum, the key informant survey is a useful measure for assessing the capacity and performance of local systems of care. The information provided by the survey should be valuable to any community striving to meet the needs of persons with a chronic mental illness.

\section{REFERENCES}

Aiken, L., Somers, S. \& Shore, M. (1987). Private foundations in health affairs: a case study of the development of a national initiative for the chronically mentally ill. American Psychologist, 41, 1290-1295.

Attkisson, C., Hargreaves, W., Horowitz, M. \& Sorensen, J. (1978). Evaluation of human service programs. San Francisco, CA: Academic Press.

Babigian, H \& Marshall, P. (1989). Rochester: a comprehensive capitation experiment. In D. Mechanic \& L. Aiken (Eds.), New directions for mental health services: Vol 43. Paying for services: Promises and pitfalls. San Francisco, CA: Jossey-Bass. 
Bachrach, L. (1980). Overview: model programs for chronic mental patients. American Journal of Psychiatry, 137, 1023-1031.

Dickey, B. \& Goldman, H. (1986). Public care for the chronically mentally ill: Financing operating costs, issues and options for local leadership. Administration in Mental Health, 14(2), 63-77.

Goldman, H., Lehman, A., Morrissey, J., Newman, S., Franks, R., \& Steinwachs, D. (1990). Evaluating the Robert Wood Johnson Program on Chronic Mental Illness. Hospital \& Community Psychiatry, 41(11), 1217-1221.

Goldman, H., Morrissey, J., \& Ridgely, MS. (1990). The mental health authority: Preliminary observations from the site-level evaluation of the Robert Wood Johnson Foundation Program on Chronic Mental Illness. Hospital \& Community Psychiatry, 41(11), 1222-1230.

Grusky, I. \& Tierney, K. (1988). Evaluating the effectiveness of countywide mental health care systems. Working paper Number 5. Los Angeles, CA: University of California.

Mechanic, D. \& Aiken, L. (1987). Improving the care of patients with chronic mental illness. New England Journal of Medicine, 317, 1634-1638.

Morris, R. \& Hirsh-Leschoier, I. (1978). Service integration: real versus illusory solutions to welfare dilemmas. In R. Sarri \& Y. Hasenfeld (eds.), The management of human services. New York: Columbia University Press.

Morrissey, J., Bartko, T., Calloway, M., Ridgely, S., Goldman, H., \& Paulson, R. (November, 1992). System change and centralization of authority during the Robert Wood Johnson Program on Mental Illness. Paper presented at the annual meeting of the American Public Health Association, Washington, D.C.

Morrissey, J., \& Tausig, M. \& Lindsey, M. (1985). Network analysis methods for mental health service system research: $A$ comparison of two community support systems. NIMH Series BN No. 6, DHHS Pub. No. (ADM) 85-1383. Washington, DC: U.S. Government Printing Office.

Morrissey, J., Tausig, M. \& Lindsey, M. (1986). Interorganizational networks in mental health systems: assessing community support systems for the chronically mentally ill. In W. Scott and B. Black (Eds.), The organization of mental health services: Societal and community systems. Beverly Hills, CA: Sage.

Program for the Chronically Mentally Ill. (1985). Program Announcement. Princeton, NJ, The Robert Wood Johnson Foundation.

Shore, M. \& Cohen, M. (1990). Creating new systems of care: the Robert Wood Johnson Foundation program on chronic mental illness. Hospital and Community Psychiatry 41(11), 1212-1216.

Sieber, S. (1973). The integration of fieldwork and survey methods. The American Journal of Sociology, 78(6):1335-1359.

Silverman, M., Ricci, E., \& Gunter, M. (1990). Strategies for increasing the rigor of qualitative methods in evaluation of health care programs. Evaluation Review, 14, 57-74.

Stroul, B. (1988). Community support systems for persons with long-term mental illness: Questions and answers. Rockville, MD: Community Support Program, National Institute of Mental Health.

Tessler, R. \& Goldman, H. (1982). The chronically mentally ill: Assessing community support programs. Cambridge, MA: Ballinger.

Turner, J. \& TenHoor, W. (1978). The NIMH community support program: Pilot approach to a needed social reform. Schizophrenia Bulletin, 4(3), 319-408.

Wilson, N., Davis, E.C., Shern, D.L. \& Cummins, K.R. (1988). The Denver CMI initiative evaluation, study 1: The key informant and attitude survey. Denver, CO: Colorado Division of Mental Health.

Walsh, A. \& Leigland, J. (1986). Public authorities and mental health programs. New York: Institute for Public Administration. 